\title{
A Unified Geometric Approach for Inverse Kinematics of a Spatial Chain with Spherical Joints
}

\author{
Li Han and Lee Rudolph
}

\begin{abstract}
Conventionally, joint angles are used as parameters for a spatial chain with spherical joints, where they serve very well for the study of forward kinematics (FK). However, the inverse kinematics (IK) problem is very difficult to solve directly using these angular parameters, on which complex nonlinear loop closure constraints are imposed by required end effector configurations. In a recent paper, our newly developed anchored triangle parameters were presented and shown to be well suited for the study of IK problems in many broad classes of linkages. The focus of that paper was the parameterization of non-singular solutions; among many specific types of IK problems, only one, that of a spatial chain with spherical joints imposing 5 dimensional constraints, was developed in detail.

Here we present a unified approach to the solutions of that and two other types of IK problems. The critical concepts in our approach-the geometric formulation in anchored triangle parameters, and the application of loop deformation spacesare general for all IK problems, and especially useful for redundant systems. For the three IK problems addressed in this paper, we demonstrate convexity properties of the set of IK solutions. We also give detailed descriptions of the parameterization of singular deformations. Similar ideas apply readily to linkages involving multiple loops.
\end{abstract}

\section{OVERVIEW}

Kinematics is fundamental in the study of linkage systems. This has long been known in robotics, where kinematics analysis, robot design, motion planning and trajectory control all involve kinematical issues. More recently, kinematics has been generalized and applied to the study of protein conformations. A key, yet somewhat un-emphasized, issue in the study of kinematics is the choice of parameters. By far the most commonly used kinematic parameters are joint parameters [1], including angles for rotational joints, translational displacements for prismatic joints, and related twists [2]. For linkages, such joint parameters are a natural default since they correspond directly to the actuation of the joints and are well suited for forward kinematics (FK) computation (e.g., by taking the product of link transformation matrices). In contrast with FK, inverse kinematics (IK) problems are usually very difficult to solve in joint parameters: typically the dependence of the end effector configurations on joint parameters (particularly for rotational joints) is highly nonlinear. Yet past research has led to a large body of impressive results; a small set of representative work appears in [1], [2], [3], [4], [5], [6], [7], [8], [9], including recent formulations of IK problems using distance constraints and vector equations.

Patent pending.

L. Han and L. Rudolph are with the Department of Mathematics and Computer Science, Clark University, Worcester, MA 01610, USA [1han, lrudolph]@clarku.edu
We recently introduced a new set of linkage parameters tailored to the study of inverse kinematics. These anchored triangle parameters consist of certain inter-joint distances (called diagonal lengths) and certain triangle orientation parameters (signs or dihedral angles); they were described in some detail for serial chains with spherical joints in space or revolute joints in the plane in [10], and for closed planar chains with revolute joints in [11]. It was shown that for the linkages under study the addressed IK problem can be formulated exactly, not approximately, as a set of linear inequalities in the anchored triangle parameters. In addition, for a spatial chain with $\tilde{n}$ spherical joints and generic link lengths, the solution set for the addressed IK problem under this new parameterization is the product of an $\tilde{n}-3$ dimensional convex polyhedron and an $\tilde{n}-2$ dimensional torus; insofar as a torus becomes convex (a cube) when it is cut open, this product can be considered "practically convex". (Extra care is needed for singular configurations.)

The inverse kinematics problem addressed in [10] is formulated with respect to the required positions of the two end points of the last link. Such a formulation is equivalent to the required end effector transformation in the planar case, but slightly different in the spatial case. More specifically, in space the two-endpoint-position constraint is 5-dimensional: it constrains the last joint's position in space (3-dimensional constraint) and the penultimate joint's position on the sphere centered at the last joint with radius the last link length (2-dimensional constraint). On the other hand, the common end-effector constraint in space imposes a 6-dimensional constraint, and the encoding of the sixth constraint in the new parameters is not addressed in [10]; nor do [10], [11] give many details on how to deal with singular configurations, especially super-singular configurations (to be explained later). Yet singular configurations play an important role in reconstructing the global topological structure of the solution set, especially for planar chains with revolute joints [11].

In this paper, we address these issues and study three types of IK problems for a serial spatial chain consisting of $\tilde{n}$ rigid links connected by spherical joints. IK Problem 1 is commonly called the "reaching" problem and poses a constraint on the position of the tip of the chain; IK Problem 2 is that studied in [10]; and IK Problem 3 imposes a 6 dimensional constraint on the chain similar to the constraints imposed by a required configuration of the last link frame. We present a unified geometric approach based on a critical concept of the deformation space of a loop. This new geometric formulation as well as the concept of the deformation space of a loop are general for all IK problems and are 
especially useful for redundant systems. In addition, for the three IK problems addressed in this paper, the anchored triangle parameterization is shown to make the set of inverse kinematics solutions piecewise convex. We also describe in detail the parameterization of singular deformations. Similar ideas apply readily to linkages involving multiple loops.

We would like to emphasize that a linkage with spherical joints can be used to model points in space under distance constraints, where the points could be mathematical points, chemical atoms, environmental landmarks for robot localization and/or mapping, or even (mini-)robots in a swarm collaborating on a task like area surveillance and coverage. See [12] for a coarse-grain protein model as a chain of alpha-carbons connected by spherical joints. While a robot linkage generally does not have many joints and is not overly redundant, the numbers of points in those point systems could be quite large; thus explicit parameterization of the deformation spaces and configuration spaces may be particularly useful in the study of those systems.

\section{AdDRESSED PRoblems}

For a spatial chain with $\tilde{n}$ spherical joints, denote by $\tilde{P}(j)$, $j=0, \ldots, \tilde{n}$, the end points of its links, including the joints and the tip of the last link. With a slight abuse of notation, we also write $\tilde{P}(j)$ for the coordinate of the point $\tilde{P}(j)$ with respect to some fixed frame. Let $\tilde{l}(j)>0$ denote the length of the link from $\tilde{P}(j)$ to $\tilde{P}(j+1), j=0, \ldots, \tilde{n}-1$. Similar to [10], we make the simplifying assumption that a chain configuration is completely specified by its joint positions, i.e., a link has no structure other than its length and the locations of its endpoints. Following common approaches taken in the study of IK, we do not consider system constraints such as joint limits and interference between links.

In this paper, we study three types of inverse kinematics problems, specified by increasingly stringent requirements on the end link configurations. In each problem, we assume that the base joint $\tilde{P}(0)$ is fixed.

- IK Problem 1 requires just the chain tip $\tilde{P}(\tilde{n})$ to be in a specified position.

- IK Problem 2 requires the last link, with endpoints $\tilde{P}(\tilde{n}-1)$ and $\tilde{P}(\tilde{n})$, to be in a specified position.

- IK Problem 3 requires the last link to be in a specified position and the second to last link, with endpoints $\tilde{P}(\tilde{n}-2)$ and $\tilde{P}(\tilde{n}-1)$, to form a specific angle $\theta_{\text {req }}$ with a given vector $v_{\text {req }}$.

Problem 1, commonly called the "reaching problem", imposes a 3-dimensional constraint, making the expected dimension of its solution space $2 \tilde{n}-3$. The problem addressed in [10] is Problem 2; it imposes a 5-dimensional constraint, so its solution space should generally be $(2 \tilde{n}-5)$ dimensional. Problem 3 imposes a 6-dimensional constraint, and thus is similar to IK problems specified by required configurations of the local frame of the last link, which has 6 degrees of freedom: indeed, the constraints imposed on $\tilde{P}(\tilde{n}-2), \tilde{P}(\tilde{n}-1)$ and $\tilde{P}(\tilde{n})$ in Problem 3 can be used to define a local frame for the last link.

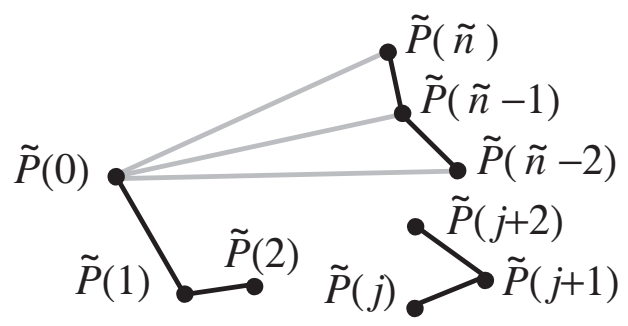

Fig. 1. Physical links (in black) and virtual links (in gray) in IK problem 3.

It follows from [13], [14] that, for each of IK Problems 13 , the solution space is an algebraic variety contained in the joint angle space (which can be identified with a $2 \tilde{n}$ dimensional torus $\left(S^{1}\right)^{2 \tilde{n}}$ ), and that for generic link lengths this algebraic variety is a manifold of the appropriate dimension embedded in $\left(S^{1}\right)^{2 \tilde{n}}$. Manifolds are a conceptually simple class of spaces, characterized by the existence at each point of a local coordinate system of real parameters that biuniquely describe all nearby points; algebraic varieties, by contrast, may contain points near which there is no biunique parametrization by any number of real parameters. Although manifolds are characterized by local Euclidean coordinate systems, a manifold per se has no obvious coordinates.

\section{Our Results and Solving Strategies}

To solve IK Problems 1-3, let us draw the virtual links from the base joint to the joints with constrained positions: $\tilde{P}(\tilde{n})$ in IK Problem 1, $\tilde{P}(\tilde{n})$ and $\tilde{P}(\tilde{n}-1)$ in IK Problem 2, and $\tilde{P}(\tilde{n}), \tilde{P}(\tilde{n}-1), \tilde{P}(\tilde{n}-2)$ in IK Problem 3. (The virtual link $\tilde{P}(0) \tilde{P}(\tilde{n}-2)$ will be explained later.) Fig. 1 illustrates IK Problem 3, with irrelevant features suppressed. Deferring the details a little bit, we see that these virtual links complement the physical links and lead to virtual loops. Our key idea in solving all these IK problems is to generate configurations of loops with appropriate link lengths, without any regard for the positions and orientations of the loop, and then to use the rigid transformations to put the constrained joints (such as $\tilde{P}(0)$ and $\tilde{P}(\tilde{n})$ in IK Problem 1) at the required positions.

In this section, we first formalize the notation of system deformations (which can be said to describe "configurations without regard to the position and location of the system as a whole") and then derive the solutions of IK Problems 13 using this concept. We then describe the parameters for the loop deformations and the geometry of the deformation space in the next two sections. In contrast to paper [10], we will explicitly consider the singular cases and solve multiple IK problems in the unified approach using the concept of deformation space.

\section{A. Deformation Space}

The configuration of a multi-object system can be described by the configuration of the objects with respect to a local frame, together with a transformation from the local frame to a fixed reference frame. Unlike a rigid body which has fixed local coordinates for all points, a multi-body 


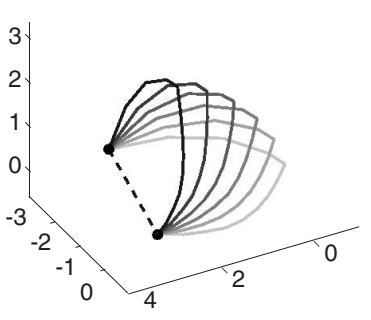

(a)

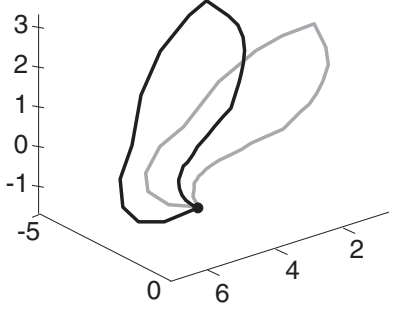

(b)
Fig. 2. Examples of IK problem 1 solutions. (Different solutions are drawn in different gray shades.) (a) General case: 6 solutions generated by rotating one deformation of a virtual loop about the virtual link (shown in a dashed line) defined by the IK problem. (b) Special case: two solutions generated by rotating one deformation of a loop about the base point.

system has different local configurations. Thus a configuration of a multi-body system is described by a rigid body transformation together with a deformation of the system that describes the relative configurations of the objects in the system. More specifically, two configurations have the same deformation if there exists a rigid transformation that moves the system from one configuration to the other. Mathematically, we can define an equivalence relation in the configuration space by the group of all rigid motions in the appropriate Euclidean space and define the the set of all deformations (or deformation space, DSpace for short) of a system to be the quotient space of its configuration space over the group of rigid motions. ${ }^{1}$

$$
\text { DSpace }=\text { CSpace } / R M
$$

As an example, a closed chain with 3 links of fixed lengths (i.e., a triangle) has a trivial deformation space consisting of only one deformation. So does a system consisting of only one rigid body. But the deformation space of a kinematic chain could be complicated with deformations facilitated by the joint degrees of freedom, and restricted by constraintse.g., fixed link lengths and, for closed chains, the loop closure constraint-independent of rigid motions and so effectively defined on DSpace of the chain.

\section{B. Solutions for IK Problems 1 and 2}

To simplify our notation, we will call a chain (loop) with $\tilde{n}$ spherical joints an $\tilde{n} S$ chain (loop). We will also denote the set of solutions for IK Problem $k$ by $I K k$ for $k=1,2,3$. Note that the set of IK solutions for a redundant manipulator is called a "self-motion manifold" [2], a concept related to but different from deformation space. For the linkages under study, the relationship between self-motion manifolds and deformation spaces will become clear in this section.

For IK Problem 1, the virtual link $\tilde{P}(0) \tilde{P}(\tilde{n})$ and physical links $\operatorname{link}(0), \ldots, \operatorname{link}(\tilde{n}-1)$ form an $(\tilde{n}+1) S$ loop, to be called Loop1. Denote its deformation space by DSpace1. Since deformations are "free flying" configurations in the Euclidean space, we can begin to pin the loop deformations

\footnotetext{
${ }^{1}$ The term deformation space (DSpace) is coined in the spirit of configuration space (CSpace) and stems from our impression that deformation is a commonly used word for relative configurations and motions of components within a system.
}

down by fixing the positions of $\tilde{P}(0)$ and $\tilde{P}(\tilde{n})$. In case these required positions are distinct, after a deformation is pinned down, its remaining degree of freedom is rotation about the axis passing through $\tilde{P}(0), \tilde{P}(\tilde{n})$. It is straightforward to prove that in this case the set of all IK solutions is $\left\{g\left(\tilde{P}(0), \overline{\tilde{P}(0) \tilde{P}(\tilde{n})}, \tau_{1}\right) \mathbf{D} \mid \mathbf{D} \in\right.$ DSpace 1$\}$, where $g\left(\tilde{P}(0), \tilde{P}(0) \tilde{P}(\tilde{n}), \tau_{1}\right)$ represents a rigid transformation needed to move a deformation $\mathbf{D}$ to the required $\tilde{P}(0), \tilde{P}(\tilde{n})$, and $\tau_{1}$ is a rotational angle. Since there is no constraint on $\tau_{1}$, IK 1 can be identified with $S^{1} \times D$ Space 1 in this case. In the remaining case, when $\tilde{P}(\tilde{n})==\tilde{P}(0)$, we need to deal with an $\tilde{n} S$ loop having link lengths $\tilde{l}(0), \ldots, \tilde{l}(\tilde{n}-1)$. Denote the deformation space of this loop by $D S p a c e 1 S$. Here the set of IK solution is the set of all chain configurations in which the loop can rotate freely about the point $\tilde{P}(0)$, and IK1 can be identified with $S O(3) \times D$ Space $1 S$ in this case. We summarize the solution for IK Problem 1 in (2) and show some examples in Fig. 2.

$$
I K 1= \begin{cases}S^{1} \times D \text { Space } 1 & \text { if } \tilde{P}(\tilde{n}) \neq \tilde{P}(0), \\ S O(3) \times D \text { Space } 1 S & \text { otherwise }\end{cases}
$$

Note that the product notation " $x$ " in (2) is not entirely rigorous. For example, in case $\tilde{P}(\tilde{n}) \neq \tilde{P}(0)$ it is possible for the link lengths to be such that the loop can degenerate into a single line segment, and then all rotations about the axis $\tilde{P}(0) \tilde{P}(\tilde{n})$ generate one single configuration. To restore rigor, we might take the symmetry of loop deformations into account, in a way similar to the symmetry subgroups of rigid motions determined by object shapes as in [15]; mathematically, this is tantamount to describing $I K 1$ as a union of fiber bundles (cf. [16]). However, we will use the product " $x$ " notation in this paper for the sake of simplicity.

The solution for IK Problem 2 can be similarly derived. It is given in (3), where DSpace 2 denotes the deformation space of an $\tilde{n} S$ loop with link lengths $\tilde{l}(0), \ldots, \tilde{l}(\tilde{n}-2)$, and $|\tilde{P}(0) \tilde{P}(\tilde{n}-1)|$ when $|\tilde{P}(0) \tilde{P}(\tilde{n}-1)| \neq 0$, while DSpace $2 S$ denotes the deformation space of the $(\tilde{n}-1) S$ loop with link lengths $\tilde{l}(0), \ldots, \tilde{l}(\tilde{n}-2)$. (The solution given in [10] corresponds to the case of $\tilde{P}(\tilde{n}-1) \neq \tilde{P}(0)$.)

$$
I K 2= \begin{cases}S^{1} \times D \text { Space } 2 & \text { if } \tilde{P}(\tilde{n}-1) \neq \tilde{P}(0), \\ S O(3) \times D \text { Space } 2 S & \text { otherwise }\end{cases}
$$

\section{Solution for IK Problem 3}

The third IK problem needs more work than the other two problems. Here the link $\tilde{P}(\tilde{n}-1) \tilde{P}(\tilde{n}-2)$ needs to form a specified angle $\theta_{\text {req }}$ with a specified vector $v_{\text {req. }}$. To satisfy this constraint alone, given a segment of length $\tilde{l}(\tilde{n}-2)$ making angle $\theta_{\text {req }}$ with the linear axis $A\left(\tilde{P}(\tilde{n}-1), v_{\text {req }}\right)$ defined by point $\tilde{P}(\tilde{n}-1)$ and the direction $v_{\text {req }}$, for each angle $\phi$ we can produce a link $\tilde{P}(\tilde{n}-1) \tilde{P}(\tilde{n}-2)$ by rotating the segment by $\phi$ about $A\left(\tilde{P}(\tilde{n}-1), v_{\text {req }}\right)$. Thus the union of all possible configurations of this link is a right circular cone in the Euclidean workspace, on which the set of all possible positions of $\tilde{P}(\tilde{n}-2)$ is a cross-sectional circle, as illustrated in Fig. 3(a). So this constraint alone imposes some restrictions on possible $\tilde{P}(\tilde{n}-2)$ positions as well as the length $v l(\tilde{n}-2)$ of the virtual link $\tilde{P}(0) \tilde{P}(\tilde{n}-2)$. 


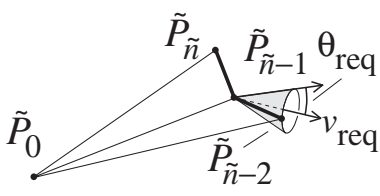

(a)

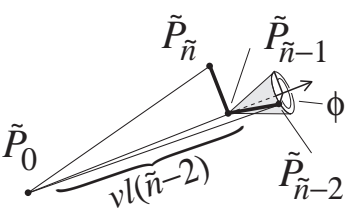

(b)

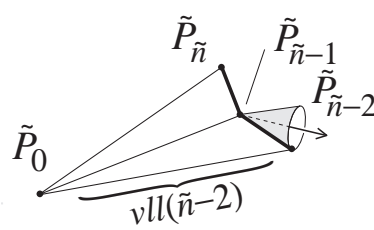

(c)

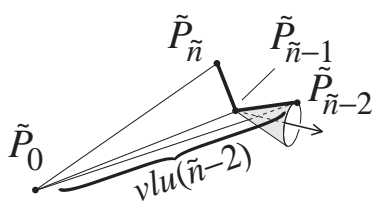

(d)

Fig. 3. Any IK problem 3 specification determines a circle for possible positions of $\tilde{P}(\tilde{n}-2)$. (a) General case. (b) Special case: the virtual link $\tilde{P}(0) \tilde{P}(\tilde{n}-1)$ is coaxial with $v_{\text {req }}$ and the virtual link length $v l(\tilde{n}-2)$ is constant. (c), (d): cis and trans forms of the general case, determining the minimum and maximum values of the virtual link length $v l(\tilde{n}-2)$.

We now describe this in more detail, again considering two cases. In the special case, illustrated in Fig. 3(b), $\tilde{P}(0)$ is on $A\left(\tilde{P}(\tilde{n}-1), v_{\text {req }}\right)$, so that the length $v l(\tilde{n}-2)$ is fixed. Here, denote the deformation space of an $(\tilde{n}-1) S$ loop with link lengths $\tilde{l}(0), \ldots, \tilde{l}(\tilde{n}-3), v l(\tilde{n}-2)$ by DSpace $3 S$. For each loop deformation, we must put $\tilde{P}(0)$ at the fixed location and $\tilde{P}(\tilde{n}-2)$ on the circle dictated by the IK3 problem specification as just described. Since $\tilde{P}(\tilde{n}-2)$ could be any point on the circle, we can use one angular parameter to parameterize its position on the circle. Furthermore, a loop with its virtual link $\tilde{P}(0) \tilde{P}(\tilde{n}-2)$ attached to the required location can still freely rotate about the virtual link. So we can use another angular parameter for this rotational freedom. Thus the set of IK solutions in this case can be described as follows.

$$
I K 3=\left(S^{1}\right)^{2} \times D S p a c e 3 S \text {, if } \tilde{P}(0) \in A\left(\tilde{P}(\tilde{n}-1), v_{\text {req }}\right)
$$

In the general case, $\tilde{P}(0) \notin A\left(\tilde{P}(\tilde{n}-1), v_{\text {req }}\right)$ and so a unique plane passes through $\tilde{P}(0)$ and $A\left(\tilde{P}(\tilde{n}-1), v_{\text {req }}\right)$. This plane intersects the circle of the possible positions of $\tilde{P}(\tilde{n}-2)$ (just considering the IK requirements) at two points, corresponding to the minimal value $\operatorname{vll}(\tilde{n}-2)$ and maximal value $v l u(\tilde{n}-2)$ of $|\tilde{P}(0) \tilde{P}(\tilde{n}-2)|$ (see Figs. 3(c) and 3(d)). For each $v l(\tilde{n}-2)$ in the open interval $(v l l(\tilde{n}-2), v l u(\tilde{n}-2))$, there correspond exactly two feasible positions for $\tilde{P}(\tilde{n}-2)$, one on each side of the plane, that are also consistent with the known positions of $\tilde{P}(0)$ and $\tilde{P}(\tilde{n}-1)$ and the required angle $\theta_{\text {req }}$ between $v_{\text {req }}$ and $\tilde{P}(\tilde{n}-1) \tilde{P}(\tilde{n}-2)$; we can label one side of the plane + and the other - , and then represent the side information specifying the position of $\tilde{P}(\tilde{n}-2)$ by $\epsilon \in S^{0}=\{+,-\}$. For the two extreme distance values $\{\operatorname{vll}(\tilde{n}-2), \operatorname{vlu}(\tilde{n}-2)\}$, $\tilde{P}(\tilde{n}-2)$ has only one feasible position, which we represent by $\{+\}$ or $\{-\}$ indifferently (although it can be argued that an entirely distinct label, e.g. 0 , might be more appropriate).

Consider a virtual loop with link lengths $\tilde{l}(0), \ldots, \tilde{l}(\tilde{n}-3), \quad v l(\tilde{n}-2)$, allowing $v l(\tilde{n}-2)$ to change between $\operatorname{vll}(\tilde{n}-2)$ and $\operatorname{vlu}(\tilde{n}-2)$, and denote its deformation space by DSpace 3 . Based on the above description, a value of $v l(\tilde{n}-2)$ in its range together with a sign label $\epsilon$ determines one position of $\tilde{P}(\tilde{n}-2)$, whence the constraints in IK Problem 3 reduce to the same constraints as in IK Problems 1 and 2, and the solution can be generally written as $S^{1} \times D$ Space 3 Slice, where DSpace 3 Slice is the slice of DSpace 3 in which $v l(\tilde{n}-2)$ is assigned some particular value. Taking the side information into account, and with a slight abuse of notation, we can say that, so long as point $\tilde{P}(0)$ is not on $A\left(\tilde{P}(\tilde{n}-1), v_{\text {req }}\right)$, the space IK3 can be written as follows.

$$
I K 3=S^{1} \times S^{0} \times D \text { Space } 3 \text {, if } \tilde{P}(0) \notin A\left(\tilde{P}(\tilde{n}-1), v_{\text {req }}\right)
$$

\section{Loop Deformation Parameters}

We now determine the deformation space of a general loop with $n$ spherical joints, i.e. an $n S$ loop for short. We will refer to the loop joints and link lengths by $P(j)$ and $l(j), j=0, \ldots, n-1$. Once the deformation space of a general $n S$ loop is determined, we can apply the results to the appropriate virtual loops arising from IK Problems 1-3. We will also allow the last link length $l(n-1)$ to have a range with known lower bound $l l(n-1)$ and upper bound $l u(n-1)$, to accommodate IK Problem 3 .

For our parameters, we will frequently refer to some $j^{\text {th }}$ entity (such as joint, link, etc.) counted from joint $i$, which should have index $(i+j) \bmod n$ to model the cyclic nature of the loop joint and link indices. To simplify the description, we write $(i ; j)$ for $(i+j) \bmod n$.

\section{A. Anchored triangle parameters for nonsingular deforma- tions}

Following the ideas in [10], we use a joint $P(a)$ as an anchor (for defining the parameters, not for restricting the positions of the loop). In general we call an object "anchored" if it includes $P(a)$. For $j=1, \ldots, n-1$, we call the vector $P(a) P(a ; j)$ an anchored diagonal of the loop and denote it $\overrightarrow{\operatorname{diag}}(a, j)$. Note that the anchored diagonals $P(a) P(a ; 1)$ and $P(a) P(a ; n-1)$ are also links of the loop. For $j=1, \ldots, n-2$, we denote by $\operatorname{Tr} i(a, j)$ the anchored triangle with vertices at joints $P(a), P(a ; j)$, and $P(a ; j+1)$; one edge of $\operatorname{Tr}(a, j)$ is $\operatorname{link}(a ; j)$ and the others are anchored diagonals $\overrightarrow{\operatorname{diag}}(a, j)$ and $\overrightarrow{\operatorname{diag}}(a, j+1)$. (See Fig. 4(a).)

For a given deformation, $\operatorname{Tr} i(a, j)$ is degenerate (i.e., reduces to an anchored line segment) if and only if its vertices are collinear, which can happen in two distinct ways: either $\operatorname{Tr} i(a, j)$ has three distinct but collinear vertices, or $\operatorname{Tr} i(a, j)$ has exactly two distinct vertices, in which case we call it doubly degenerate. (Since all link lengths are positive, no anchored triangle can reduce to a point.) Note that $\operatorname{Tr} i(a, j)$ is doubly degenerate in a given deformation if and only if one of $P(a ; j), P(a ; j+1)$ coincides with $P(a)$, so that $\operatorname{Tr} i(a, j-1)$ or $\operatorname{Tr} i(a, j+1)$, respectively, is also doubly degenerate. We call a loop deformation singular for 


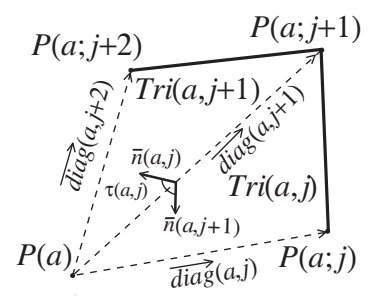

(a)

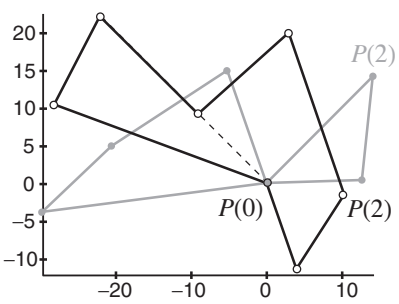

(b)
Fig. 4. Loop deformations. (a) Anchored triangle parameters: diagonal lengths and dihedral angles. (b) Two deformations of a 7-bar loop: neither is singular for $P(2)$, but both are singular for $P(0)$ and the one in gray is also supersingular for $P(0)$ (and $P(3)$ ).

the given anchor $a$ if it has at least one degenerate anchored triangle, and super-singular, again for that anchor, if it has at least one doubly-degenerate anchored triangle. Fig. 4(b) shows two deformations of a certain 7-bar loop: both singular for the anchor $P(0)$, that drawn in gray is supersingular, while that drawn in black is singular but not super-singular, with the collinearity of two adjacent joints with $P(0)$ shown in a dashed line. Note that neither deformation is singular for, e.g., the anchor $P(2)$. We will get back to the role of anchor choice in the next subsection. Here just note that for our new parameterization and a given deformation, whether the deformation is singular or not depends on the choice of anchor. In this subsection, we focus on the set of non-singular deformations with respect to a given choice of anchor $P(a)$ and denote this set by $D N S(a)$.

In a sense, the new parameters for DSpace are the triangles $\operatorname{Tr} i(a, j)$ themselves, as embedded in space modulo a single rigid motion (applied to all at once). We define more conventional parameters from them as follows (see Fig. 4(a)).

Definitions 1: Anchored Triangle Parameters

- diagonal lengths: $\overline{\mathbf{r}}(a)=(r(a, 1), \ldots, r(a, n-3)) \in$ $\mathbf{R}^{n-3}$, where $r(a, j)=\|P(a) P(a ; j+1)\|$ for $j=$ $1, \ldots, n-3$.

- dihedral angles: $\bar{\tau}(a)=(\tau(a, 1), \ldots, \tau(a, n-3))$, where the dihedral angle $\tau(a, j)$ is the signed angle for rotating the normal of $\operatorname{Tr} i(a, j)$ to that of $\operatorname{Tr} i(a, j+1)$.

- changeable link length: only $l(n-1)$ for now to model the changeable length of the virtual link $\tilde{P}(0) \tilde{P}(\tilde{n}-2)$ in IK Problem 3. For IK Problems 1 and 2, just make the lower and upper bounds of this link length equal to the fixed link length $l l(n-1)=l u(n-1)=l(n-1)$.

Clearly for any deformation in $D N S(a)$, all these parameters are well defined. To see that these parameters can indeed be used to determine the loop deformations, note that for a given set of valid diagonal lengths and dihedral angles, the lengths of the links and anchored diagonals completely determine the shapes of the anchored triangles, and the dihedral angles determine the relative orientations of each pair of adjacent anchored triangles. Then we can use an anchored triangle (more generally, any set of three noncollinear points) as a reference triangle and put it anywhere in space. Next, we place its neighboring triangle(s) using their shape(s) and relative orientation(s). We continue this iterative process until all triangles have been placed in completely uniquely defined locations, leading to a well defined loop deformation. (The detailed formula given in [10] to carry out this computation assumes that the anchor is $P(0)$, but it is easily modified to use any other anchor.) This process just described essentially proves the following theorem, which means that the anchored triangle parameters are the local coordinates of the set of all non-singular loop deformations (again with parameters and deformation singularity defined with respect to the same anchor).

Theorem 1: The mapping between the set of non-singular deformations in $D N S(a)$ and their corresponding anchored triangle parameters $(\overline{\mathbf{r}}(a), \bar{\tau}(a), l(n))$ is one-to-one.

\section{B. Anchored triangle parameters for singular deformations}

In terms of a specified anchor, the diagonal lengths are well defined for all deformations; but the dihedral angles are only well defined for non-singular deformations, since the degenerate anchored triangles in a singular deformation do not have well-defined normals. So our deformation parameters must be modified to handle singular deformations. We have two approaches to address this issue.

In the first approach, we continue to use the same anchor and the anchored triangles. In this approach, the modification is simple for a deformation that is singular but not supersingular. Assume $\operatorname{Tr} i(a, j)$ is such a triangle. We can choose any unit vector in the well-defined plane perpendicular to the line segment of the degenerate triangle as $\bar{n}(a, j)$. In practice, we can make some convenient choice such as by setting $\bar{n}(a, j)$ to the normal of one of its neighboring triangles $\operatorname{Tr} i(a, j-1)$ and $\operatorname{Tr} i(a, j+1)$ when applicable. However, notice that for a given singular deformation, different choices of $\bar{n}(a, j)$ will lead to different values of the dihedral angles involving this triangle. Another way of dealing with the situation is to define one dihedral angle for every two adjacent non-singular anchored triangles, instead of for every two adjacent triangles. With this alternative definition, there will be fewer parameters for singular deformations than for non-singular deformations. The reduced set of parameters, however, better reflects the intrinsic "instantaneous" deformational degrees of freedom (the dimension of the tangent space at the deformation) at singular deformations. Note that for two adjacent non-singular triangles, rotating one about their shared diagonal will lead to new deformations of the pair. But when at least one of the two adjacent anchored triangles is singular, the relative torsional rotation between the pair does not lead to any new deformations of the pair. So each singular anchored triangle loses one torsional degree of freedom, which will be captured by the alternative definition of dihedral angles. However, we would like to emphasize that for the convenience of computation as afforded by a constant number of parameters, it is okay to use the original definitions of dihedral angles for singular but not super-singular deformations, with the proper understanding of the dihedral angles involving singular anchored triangles. Moreover, if we assign the normal $\bar{n}(a, j)$ of a singular anchored triangle $\operatorname{Tr} i(a, j)$ to that of its predecessor $\bar{n}(a, j-1)$, the dihedral angle $\tau(a, j-1)$, as well as other dihedral angles leading 
to singular anchored triangles, will be 0 ; non-zero dihedral angle values will effectively correspond to dihedral angles between adjacent non-singular anchored triangles.

If a configuration is super-singular, all the length- 0 diagonals correspond to joints that are coincident with the anchor. Assume that a loop deformation has $m$ length- 0 diagonals $r\left(a, j_{i}\right)=0, j_{1}<j_{2}<\cdots<j_{m}$, which means that joints $P\left(a ; j_{i}+1\right)$ and the anchor joint $P(a)$ overlap each other. Now define a sequence $P S=\left(P(a), P\left(a ; j_{1}+\right.\right.$ $\left.1), \ldots, P\left(a ; j_{m}+1\right), P(a)\right)$. Clearly the links between the successive point pairs in $P S$ define a sub-loop, and the original loop consists of these $m+1$ subloops, none of them being super-singular but all sharing the anchor joint. (Compare Fig. 4(b), in which the super-singular deformation drawn in gray gives an example of a loop with $m=1$.) Clearly each loop can freely rotate about the anchor, without violating the closure constraints of the loop. So we can model such mobility of each sub-loop, on top of the loop deformation, by $3 D$ rotation $S O(3)$. To describe the deformation of the original loop, we can use the anchored parameters for each of the $m+1$ non-super-singular subloops along with $m$ sets of $S O(3)$ parameters for the relative orientations between the subloops.

The second approach is to change anchor to a different joint. Recall that in the new parameters, whether a loop deformation is singular or not depends on the choice of anchor. As mentioned above, neither deformation shown in Fig. 4(b) is singular with respect to joint $P(2)$. This means that when $P(2)$ is used as the anchor, the anchored triangle parameters, in particular the original definition of dihedral angles, are well defined for both deformations. Of course it is possible that for a loop with given link lengths, every choice of anchor may have some loop deformations classified as (super) singular with respect to it. In that case, no choice of anchor will make it possible to use the anchored triangle parameters to parameterize the whole deformation space of the loop. But based on Theorem 1, we can consider one choice of anchor along with the corresponding anchored triangle parameters as a local coordinate chart for DSpace that covers the set of all non-singular deformations with respect to that anchor. The union of these coordinate charts

$$
D N S=\bigcup_{a=0}^{n-1} D N S(a)
$$

covers the set of loop deformations that are non-singular with respect to at least one joint. In other words, a deformation is not covered by the charts of this atlas if and only if it is singular with respect to every joint. Clearly these very special deformations, if they exist, form a set with much lower dimension than DSpace (except for trivial cases). For these special deformations, we can use the ideas described earlier such as the alternative definitions of the dihedral angles and the sub-loop concepts to derive their parameters. Another interesting idea to explore is to use a non-joint point in space as an anchor.

These two approaches complement each other and have different traits. If we know a priori some deformations in some addressed problems, such as in the case of path planning where two query deformations are given a priori, it would be generally more convenient to find a chart that covers these deformations. In this case, the second approach will probably be more helpful. On the other hand, if we need to work on deformations of required singularity with respect to a particular joint $P(i)$, it would be better to use that joint as the anchor and explicitly model the required singularity as opposed to using another anchor $P(j)$ with respect to which the required singularity does not induce singularity in the new set of anchored triangles and the constraints for maintaining the required singularity with respect to $P(i)$ are difficult to model in terms of anchored triangle parameters $(\overline{\mathbf{r}}(j), \bar{\tau}(j))$.

\section{GeOMETRY OF THE DEFORMATION SPACE}

Here we give a brief description of the deformation space of an $n S$ loop, generalizing the results given in [10]. In particular, we consider $D N S(a)$, the set of non-singular deformations with respect to anchor $P(a)$, and briefly explain that it is the product of two factor spaces, DFlip and the interior of DStretch $(a)$, as follows.

$$
\begin{aligned}
D N S(a)= & \{(\overline{\mathbf{r}}(a), \bar{\tau}(a), l(n-1)) \text { for non-sing dfms }\} \\
= & \operatorname{DFlip} \times \operatorname{Int}(\operatorname{DStretch}(a)) \\
\text { DFlip }= & \{\bar{\tau} \mid \tau(a, j) \in[-\pi, \pi], j=1, \ldots, n-3\} \\
= & \left(S^{1}\right)^{n-3} \\
\operatorname{DStretch}(a)= & \{(\overline{\mathbf{r}}(a), l(n-1)) \mid T \overline{\mathbf{r}}(a) \leq \overline{\mathbf{b}}(a), \ldots, \\
& l l(n-1) \leq l(n-1) \leq l u(n-1)\}
\end{aligned}
$$

DFlip is the set of all feasible dihedral angle values and is equivalent to an $n-3$ dimensional torus because the loop closure constraints impose no restrictions on feasible dihedral angle values. Consider the case that we have a valid loop deformation and then change the value of one dihedral angle. Such a change corresponds to flipping a subchain about an anchored diagonal such as shown in Fig. 5(a). Clearly this flipping process does not break the loop and does not change any link lengths or anchored diagonal lengths. Such an observation also shows that the anchored diagonal lengths and the anchored dihedral angles are uncoupled and that $D N S(a)$ is the product of two subspaces.

Furthermore, the loop closure constraints are linear inequality constraints on feasible anchored diagonal lengths and link lengths, since (i) any set of diagonal lengths and link lengths is feasible for forming a loop if and only if these lengths allow successful formation of all $n-2$ anchored triangles, and (ii) the side lengths of each (possibly) degenerate triangle need to satisfy the triangle inequalities. Recall that the triangle inequalities for a triangle with lengths $l 1, l 2, l 3$ are $l 1 \leq l 2+l 3, l 2 \leq l 3+l 1, l 3 \leq l 1+l 2$. Furthermore, the triangle is non-degenerate if and only if all three inequalities are strict. In our case, taking together these inequalities for $\operatorname{Tri}(a, 1), \ldots, \operatorname{Tr} i(a, n-2)$ give an explicit description of $D \operatorname{Stretch}(a)$ in terms of the link lengths $l(0), \ldots, l(n-1)$ : it is the set of solutions $(r(a, 1), \ldots, r(a, n-3))$ of the 


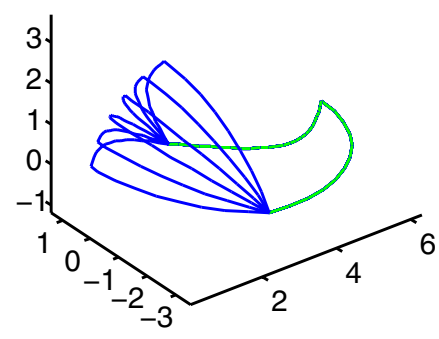

(a) Flip a subchain

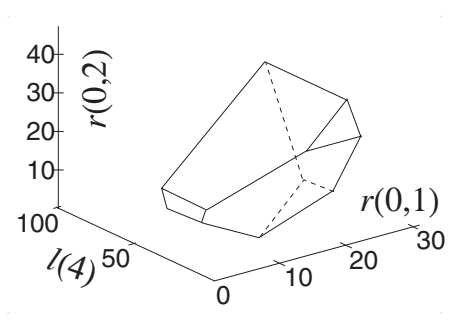

(b) $\operatorname{DStretch}(0)$

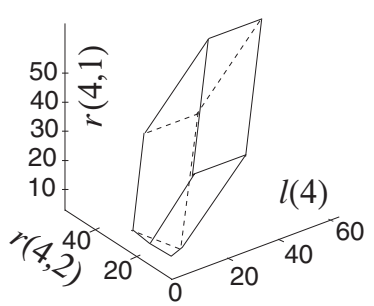

(c) DStretch(4)

Fig. 5. Loop deformations. (a) Flipping a subchain of a loop maintains loop closure and generates more deformations. (b) and (c): Two examples of $\operatorname{DStretch}(a)$ for a 5-bar loop with one link $l(4)$ having variable link lengths.

following system of linear inequalities.

$$
\begin{aligned}
& r(a, 1) \leq l(a)+l(a ; 1) \\
& -r(a, 1) \leq-|l(a)-l(a ; 1)| \\
& r(a, j)-r(a, j-1) \leq l(a ; j)) \\
& -r(a, j)+r(a, j-1) \leq l(a ; j)\} 2 \leq j \leq n-3 \\
& -r(a, j)-r(a, j-1) \leq-l(a ; j)\} \\
& r(a, n-3) \leq l(a ; n-2)+l(a ; n-1) \\
& -r(a, n-3) \leq-|l(a ; n-2)-l(a ; n-1)|
\end{aligned}
$$

Rewritten in matrix format, this system becomes $T \overline{\mathbf{r}}(a) \leq$ $\overline{\mathbf{b}}(a)$, where $\overline{\mathbf{b}}(a)$ is the vector of terms on the right hand side of the inequalities, and the $(3 n-8) \times(n-3)$ matrix $T$ has one row for each inequality in the system. The definition of DStretch(a), the set of all feasible lengths of the diagonals and $\operatorname{link}(n-1)$, as given in equation (7) includes this triangle inequality constraint along with the range constraints on $l(n-1)$. Clearly, as the intersection of half-spaces, DStretch (a) is a convex polytope. Also the link lengths are bounded in our study. So all the diagonal lengths are bounded due to the triangle inequality constraints, which means that DStretch $(a)$ is a convex polyhedron. Figs. 5(b) and 5(c) show the DStretch $(a)$ of a 5-bar loop with the first 4 links having lengths $10,12,13$, and 27 , and the last link having a length range of $[0,62]$; the anchor joint $a$ is $P(0)$ in Fig. 5(b) and $P(4)$ in Fig. 5(c). Note that for each feasible value of $l(n-1)$, the interior of the restriction of $D \operatorname{Stretch}(a)$ to its subset with the chosen $l(n-1)$ value (like a slice of DStretch $(a)$ ) corresponds to the set of non-singular deformations. The union of the interiors of these restricted slices of $D \operatorname{Stretch}(a)$, denoted by $\operatorname{Int}(\operatorname{DStretch}(a))$ (with a slight abuse of notation) in (6), consists of all valid $(\overline{\mathbf{r}}(a), l(n-1))$ for non-singular deformations.

\section{DISCUSSION}

From the previous section, we can see that the deformation space of a spatial $n S$ loop in general has dimension $2 n-6$ when all link lengths are fixed, or $2 n-5$ when the length of one link has a non-trivial range; the anchored triangle parameters describe all deformations that are non-singular for the given anchor. Referring back to equation 2 for the solution for IK Problem 1, we see that in both cases it is easy to check that the anchored triangle parameters together with the parameters for $S^{1}$ or $S O(3)$, totaling $2 \tilde{n}-3$, can serve as local coordinates for the set of non-singular IK1 solutions. Similar results also hold for IK2 and IK3 solutions.

In the same spirit in which we consider a torus $\left(S^{1}\right)^{k}$ $(k \geq 1)$ to be practically convex, we can also consider parameters for $S O(3)$ (e.g.., Euler angles) as practically convex. Then we can say that all these local coordinates for the three IK solutions sets have piecewise practically convex domains: clearly the solution sets for the first two IK problems may have only one (possibly empty) piece each; but the third solution set may have two pieces. Recall that the reachable positions for $\tilde{P}(\tilde{n}-2)$ are restricted to the circle dictated by the IK problem specification as described earlier. $\tilde{P}(\tilde{n}-2)$ is also the tip of the open sub-chain from $\tilde{P}(0)$ to $\tilde{P}(\tilde{n}-2)$ via $\tilde{P}(1)$. So its reachable positions are also restricted by this subchain and generally lying in a spherical shell bounded by two concentric spheres, with their radii determined by the link lengths $\tilde{l}(0), \ldots, \tilde{l}(\tilde{n}-3)$. Note that for a particular chain, the shell workspace of the tip $\tilde{P}(\tilde{n}-2)$ could degenerate into a sphere when the (minimal) feasible end-to-end distance of this subchain is 0 . In general, the reachable workspace of $\tilde{P}(\tilde{n}-2)$ for a given IK problem 3 is the possibly empty intersection of the circle and the shell, as shown in Fig. 6. If the intersection is indeed empty, the IK problem is not feasible. If the intersection is one arc, the IK3 solution set is not empty and has one connected component. And if the intersection has two separated arcs, the IK3 solution set has two connected components. As for the first two IK problems, they have empty solutions when the virtual "loops" have empty deformation space meaning that the virtual link lengths determined by the IK specifications are impossible to form "virtual" loop with the given link lengths. In other words, the solution sets are empty when the required positions of the end joints are out of the reach of the chain.

Note that our approach can be readily applied to the parameterization of multiple-loop systems. In particular, for a system that can be viewed as consisting of uncoupled subsystems, its deformation space is the product of the deformation spaces of the subsystems along with the relative motions between the subsystems. So for a system consisting of two chains (loops) sharing no entity, one joint or one link, its deformation space is the product of the deformation spaces of its two subsystems along with $S E(3), S O(3)$, or $S^{1}$ respectively. Note that even for two loops sharing one link, they can be viewed as two uncoupled loops for the 


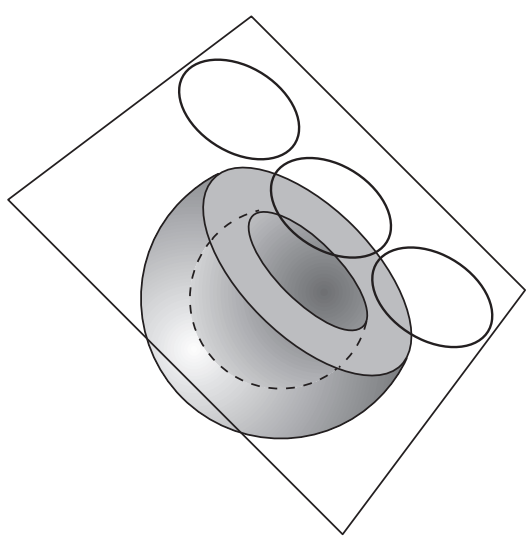

Fig. 6. The reachable workspace of $\tilde{P}(\tilde{n}-2)$ for a specific $I K$ problem 3 is the intersection of a circle (as in Fig. 3) and a possibly-degenerate spherical shell, which could be empty, two disjoint arcs or one arc.

study of their individual deformation spaces but are required to have the shared link coincident for the study of the deformations (and configurations) of the system as a whole. For two loops sharing a chain with more than one link, we can introduce a virtual link between the two ends of the shared chain and then consider three loops sharing this one virtual link.

\section{SUMMARY}

Inverse kinematics is a fundamental topic in the study of linkage systems and is considered to be very hard when formulated with respect to joint parameters especially for a chain with many joints. Our recently developed anchored triangle parameters [10], [11], consisting of anchored diagonal lengths, anchored triangle orientations (such as dihedral angles for the linkages studied here), and variable link lengths if any, are particularly well suited for many broad classes of planar and spatial linkages, including planar chains and loops with revolute joints, spatial chains and loops with spherical joints, chains with variable link lengths (which can model some prismatic joints), and some kinematic structures more complicated than a chain or single loop. What is striking is that the loop closure constraints, arising from the physical loops as well as virtual loops for IK problems, are linear inequalities in this set of geometric parameters. This new formulation renders nice geometry to the set of solutions satisfying the closure constraints and facilitate efficiently solving methods for the constraints even for chains with arbitrary numbers of joints. The parameters for a spatial chain with spherical joints were presented in the paper [10], from the perspective of solving a particular formulation of the IK problem, and focused on non-singular configurations.

In this paper, we showed a unified approach for solving three types of inverse kinematic problems, with different IK problems imposing different constraints. The IK problem addressed in [10] is also included here, but is solved in a more general and unified framework, which works for all three IK problems here and can be generalizable to general IK problems of chains with any type of joints. It is particularly useful for redundant systems and is based on the critical concept of the deformation space for a virtual loop, in order to focus on the loop closure constraints which are independent of rigid motions. We also included detailed discussions on singular deformations and explained that our approach can also be used for parameterization of deformations of linkages with multiple loops. Furthermore, we introduced the new concept of treating one choice of the anchor and the corresponding anchored triangle parameters as one local coordinate chart covering the set of deformations non-singular for that anchor, which leads to a more rigorous treatment of using the atlas of the local coordinate charts over possible choices of anchor joints to cover the set of all deformations. Part of our ongoing research is to generalize the parameterization and IK solving approaches to other (physical and virtual) linkages such as those with other (than spherical joints in space and revolute joints in the plane) and even mixed joint types as well as those with more complicated loop structures.

\section{ACKNOWLEDGMENTS}

The authors thank anonymous reviewers and editors for their helpful comments.

\section{REFERENCES}

[1] J. J. Craig, Introduction to Robotics: Mechanics and Control, 2nd Edition. Reading, MA: Addison-Wesley Publishing Company, 1989.

[2] R. M. Murray, Z. Li, and S. S. Sastry, A Mathematical Introducation to Robotic Manipulation. Boca Raton, FL: CRC Press, 1994.

[3] J.-P. Merlet, Parallel Robots. Springer, 2000.

[4] M. Mason, Mechanics of Robotic Manipulation. The MIT Press, 2001.

[5] L.-W. Tsai and A. Morgan, "Solving the kinematics of the most general six- and five-degree-of-freedom manipulators by continuation methods," ASME J. Mechanisms, Transmissions, and Automation in Design, vol. 107, pp. 189-200, 1985.

[6] M. Raghavan and B. Roth, "Inverse kinematics of the general 6r manipulator and related linkages," J. Mechanical Design, vol. 115, pp. 502-508, 1993.

[7] R. S. Rao, A. Asaithambi, and S. K. Agrawal, "Inverse kinematics solution of robot manipulators using interval analysis," ASME Journal of Mechanical Design, vol. 120, pp. 147-150, 1998.

[8] J. Porta, L. Ros, and F. Thomas, "Invese kinematics by distance matrix completion," in Proc. CK2005 (12th International Workshop on Computational Kinematics), 2005.

[9] —_, "Multiple-loop position analysis via iterated linear programming," Robotics: Science and Systems, 2006.

[10] L. Han and L. Rudolph, "The inverse kinematics of a serial chain with joints under distance constraints," in Proceedings of Robotics: Science and Systems (RSS), 2006.

[11] L. Han, L. Rudolph, J. Blumenthal, and I. Valodzin, "Stratified deformation space and path planning for a planar closed chain with revolute joints," in Proc. Seventh International Workshop on the Algorithmic Foundations of Robotics (WAFR 2006). New York: Springer, 2006.

[12] G. Song and N. M. Amato, "A motion planning approach to folding: From paper craft to protein folding," in Proc. IEEE Int. Conf. Robot. Autom. (ICRA), 2001, pp. 948-953.

[13] J. Trinkle and R. Milgram, "Complete path planning for closed kinematic chains with spherical joints," Int. J. Robot. Res., vol. 21, no. 9, pp. 773-789, 2002.

[14] R. Milgram and J. Trinkle, "The geometry of configuration spaces for closed chains in two and three dimensions," Homology Homotopy and Applications, 2002.

[15] J. Gou, Y. Chu, and Z. Li, "On the symmetric localization problem," IEEE Trans. Robot. \& Automation, vol. 14, pp. 533-40, 1998.

[16] M. Davis, "Smooth $G$-manifolds as collections of fiber bundles," Pacific J. Math., vol. 77, no. 2, pp. 315-363, 1978. 